Original Paper http://ajol.info/index.php/ijbcs http://indexmedicus.afro.who.int

\title{
Caractéristiques Socio-économiques et techniques des élevages piscicoles du Département du Mbam et Inoubou
}

\author{
Claudine Tekounegning TIOGUÉ ${ }^{1 *}$, Armand BIBOU ${ }^{2}$, Augustave KENFACK ${ }^{3}$ et \\ Joseph TCHOUMBOUÉ 3
}

'Laboratoire d'Ichtyologie et d'Hydrobiologie Appliquées, Faculté d'Agronomie et des Sciences Agricoles, Filière des métiers du Bois, de l'Eau et de l'Environnement, Université de Dschang, BP 786, Campus d'Ebolowa, Cameroun.

${ }^{2}$ Laboratoire d'Ichtyologie et d'Hydrobiologie Appliquées, Département de Productions Animales, Faculté d'Agronomie et des Sciences Agricoles, Université de Dschang, BP 222, Dschang, Cameroun.

${ }^{3}$ Laboratoire de Reproduction et de Santé Animales, Département de Productions Animales, Faculté d'Agronomie et des Sciences Agricoles, Université de Dschang, BP 188, Dschang, Cameroun.

*Auteur correspondant; E-mail : claudinetekou@gmail.com / claudinetiogue@gmail.com / claudine.tiogue@univ-dschang.org

\section{RESUME}

Le but principal de cette étude dans le Département du Mbam et Inoubou de février à juin 2019 était de fournir des connaissances générales des élevages piscicoles pour l'amélioration de leurs rendements. Un questionnaire a donc été soumis à 40 pisciculteurs dans 7 des 9 Arrondissements du Département. Les résultats obtenus révèlent que : seulement 26,39\% des 144 étangs dénombrés sont actifs. Les pisciculteurs sont à $95 \%$ des hommes de tranche d'âge majoritaire comprise entre 50 et 59 ans (40\%); de profession dominante l'agriculture $(37,50 \%)$. Bafia est l'ethnie majoritaire $(47,50 \%)$ et le christianisme est le plus rependue $(77,50 \%)$. Le niveau d'études est dominé par le supérieur (52,50\%). La main d'œuvre la plus utilisée est salariale. Les structures sont à $85 \%$ individuelles, et sont construites sur fonds personnels. Les structures sont à $95 \%$ à moins de 20 ans d'existence. La polyculture (Tilapia, silure, carpe, Kanga et Parachana) est pratiquée à $70 \%$ au sein des étangs en déblais $(82,50 \%)$ de nappes phréatiques. Les silures sont les espèces les plus élevées $(36,14 \%)$. Les alevins proviennent à $52,50 \%$ des projets et des centres d'alevinage. La fertilisation est pratiquée à 50\%, l'aliment composé rentre à $35 \%$ dans l'alimentation et les producteurs sont tous orientés vers le grossissement. Le vol $(60 \%)$ et les causes zootechniques $(25 \%)$ constituent les contraintes classiques majeures que connait la pisciculture dans cette zone. Dans ce Département l'activité piscicole est encore pratiquée de manière artisanale. A l'avenir, l'état devrait accompagner les acteurs de cette activité par de multiples formations.

(C) 2020 International Formulae Group. All rights reserved.

Mots clés : Pisciculture, caractérisation, contraintes, Région du Centre, Cameroun. 


\title{
Socio-economic and technical characteristics of fish farming in the Department of Mbam and Inoubou
}

\begin{abstract}
The main objective of this study in the Department of Mbam and Inoubou from February to June 2019 was to provide general knowledge of fish farms for the improvement of their yields. A questionnaire was therefore submitted to 40 fish farmers in 7 of the 9 Arrondissements of the Department. The results show that: only $26.39 \%$ of the 144 ponds counted are active. The fish farmers are $95 \%$ of men of majority age group between 50 and 59 years (40\%); predominantly in agriculture (37.50\%). Bafia is the majority ethnic group (47.50\%) and Christianity is the most widespread $(77.50 \%)$. The level of education is dominated by the superior $(52.50 \%)$. The most used workforce is salary. The structures are $85 \%$ individual, and are built on personal funds. $95 \%$ of structures are less than 20 years old. Polyculture (Tilapia, catfish, common carp, kanga and viper fish) is practiced at $70 \%$ in excavated ponds $(82.50 \%)$ of groundwater. Catfish are the most raised species $(36.14 \%) .52 .50 \%$ of the fingerlings come from projects and nursery centers. Fertilization is carried out at $50 \%$, the compound feed returns to $35 \%$ in the feed and the producers are all oriented towards fattening. The flight $(60 \%)$ and zootechnical causes $(25 \%)$ constitute the major classic constraints experienced by fish farming in this area. It was concluded that the fish farming activity in this area is still artisanal. In the future, the state should support the players in this activity through multiple training.
\end{abstract}

(C) 2020 International Formulae Group. All rights reserved.

Keywords: Fish farming, characterization, constraints, Center Region, Cameroon.

\section{INTRODUCTION}

Le poisson assure pour des populations pauvres des apports en nutriments essentiels tels que le fer, l'iode, le calcium, le zinc, la vitamine A et la vitamine B (WorldFish, 2005). Au Cameroun, il constitue une source majeure de protéines pour une bonne partie de la population. Il représente environ $40 \%$ de l'apport protéique d'origine animale et 9,5\% des besoins totaux pour la couche de la population rurale la moins nantie. Or, la quasitotalité du poisson consommé provient des pêches de capture $(93,4$ millions de tonnes en 2014) (FAO, 2016, 2018), qui de moins en moins sont incapables de satisfaire la demande. $\mathrm{Au}$ Cameroun, les importations de poissons ont atteint 270000 tonnes pour une demande nationale d'environ 400000 tonnes en 2013 (MINEPIA, 2015; Tomedi, 2015); or la production nationale n'est que de 1000 tonnes (Tomedi, 2015), soit un déficit de 129000 tonnes. Selon l'INS (2013), les importations se sont élevées à 181678 tonnes pour une valeur nette de 114,3 milliards de francs.

$\mathrm{La}$ filière aquacole camerounaise rencontre de nombreuses difficultés notamment : faible dispositif de formation et d'encadrement technique des pisciculteurs et acteurs du sous-secteur piscicole (Kouam, 2004); dispersion et faible organisation des acteurs, rareté et prix élevé des alevins de qualité; difficulté d'accès au crédit auprès des banques commerciales et des établissements de microfinances; absence de traditions piscicoles (Tomedi, 2015; Kenfack et al., 2019) et l'inexistence d'un tissu industriel adéquat pour la transformation des produits d'élevage (Teleu et Ngatchou, 2006 ; Kenfack et al., 2019). Selon ces derniers auteurs l'une des causes principales attribuées à cet échec a été la mise en oeuvre de politiques inadaptées.

Selon UNCHS (2001), la croissance démographique est très rapide dans la plupart des villes. Cette croissance urbaine rapide serait due à de facteurs multiples (situation économique difficile, baisse de la production vivrière, ...) qui amènent un fragment important de cette population à innover pour assurer sa survie, et qui ont contribué ainsi au développement de l'agriculture et de l'élevage urbain et périurbain (Kando, 1995). Ces élevages sont marqués par une grande diversité d'espèces (les carpes (Cyprinus carpio, Ctenopharyngodon idela), Oreochromis niloticus, Heterotis niloticus, Parachanna obscura, les poissons chats (Clarias 
gariepinus, Clarias jaensis, Heterobranchus longifilis) (Tiogué et al., 2010 ; Zango et al., 2016) et des systèmes de production sans qu'on ne témoigne un jugement raisonné de leur importance relative en termes d'effectif, de rôle socio-économique, du nombre d'acteurs concernés et d'effectif d'animaux (Kando, 1995). Quelles sont donc effectivement les caractéristiques de la pisciculture camerounaise ?

L'objectif général de cette étude est donc de caractériser l'activité piscicole dans le Département du Mbam et Inoubou au Cameroun. De manière spécifique, il est question de déterminer pour les élevages piscicoles de ce Département, les caractéristiques socio-économiques et techniques, les pratiques sanitaires et les contraintes auxquelles font face les pisciculteurs.

\section{MATÉRIEL ET MÉTHODES Zone d'étude}

L'étude s'est déroulée de février à juin 2019 dans le Département du Mbam et Inoubou, Région du Centre au Cameroun (Figure 1). Ce département se situe entre $4^{\circ}$ et $5^{\circ}$ de latitude Nord et entre $10^{\circ} 22^{\prime}$ et $11^{\circ} 30^{\prime}$ de longitude Est. Il est limité au Nord par les départements du Mbam et Kim, Noun et du Ndé, au Sud par ceux de la Lékié et la Sanaga maritime, à l'Ouest par ceux du Mkam et Ndé et à l'Est par celui de la Haute Sanaga. Il compte à ce jour 09 Arrondissements (Bafia, Bokito, Deuk, Kiiki, KonYambéta, Makénéné, Ndikiniméki, Nitoukou et Ombéssa), et couvre une superficie de $7125 \mathrm{~km}^{2}$ (Tsamo, 2008). La pluviométrie moyenne varie de 1500 à 2000 $\mathrm{mm}$. Le climat dans ce Département est de type équatorial guinéen à 4 saisons : une grande saison sèche qui va de décembre à mi-mars ; une petite saison de pluies de mi-mars à juin ; une petite saison sèche de mi-juin à août et une grande saison de pluies de septembre à novembre. La température varie entre $23{ }^{\circ} \mathrm{C}$ et $24^{\circ} \mathrm{C}$ avec un pic de $38^{\circ} \mathrm{C}$ en février, période pendant laquelle l'évapotranspiration atteint son maximum (Tsamo, 2008).

\section{Conduite de l'étude et collecte des données}

Des informations obtenues auprès des différents services publics et des partenaires au développement ont permis de déterminer les arrondissements potentiels à sillonner et de localiser un bon nombre de pisciculteurs. Par la suite, la technique de boule de neige (Wilhelm, 2014) a permis d'identifier les autres pisciculteurs dans ces différents Arrondissements une fois les premiers repérés.

L'étude a été menée dans sept (07) arrondissements du département (Bafia, Bokito, Kiiki, KonYambeta, Ndikiniméki, Nitoukou et Ombessa). Environ 56 pisciculteurs ont été identifiés dont 40 ont accepté de partager les réalités de leurs activités et 16 autres étaient indisponibles. Les informations (données) sur les éleveurs ont été obtenues à base d'un questionnaire, des échanges avec les pisciculteurs et des observations directes faites chez ces derniers. Celles-ci concernaient :

- Les caractéristiques socioéconomiques qui portaient sur l'âge, le sexe, la religion, le nombre d'enfants en charge, la situation matrimoniale, l'ethnie, le niveau d'étude, la profession, les sources de revenus et le revenu mensuel moyen des éleveurs.

-Les caractéristiques techniques et zootechniques concernaient le type d'étang, le type d'élevage, le système d'élevage, l'espèce de poisson élevé, le nombre d'étangs, la provenance des premiers animaux, intervention dans la reproduction, les structures de vidange, l'alimentation et la fréquence.

-Les informations sur la gestion sanitaire concernaient le nettoyage des étangs, la protection, l'utilisation des herbicides, présence des maladies et les méthodes de traitements.

-Des informations sur les contraintes portaient sur la perception du secteur, les difficultés rencontrées, l'implication des autorités publiques et l'accès à l'information.

\section{Analyses des données}

Les données collectées ont été analysées à l'aide de la statistique descriptive et le logiciel Microsoft Excel version 2016 a servi au traitement de ces données. 


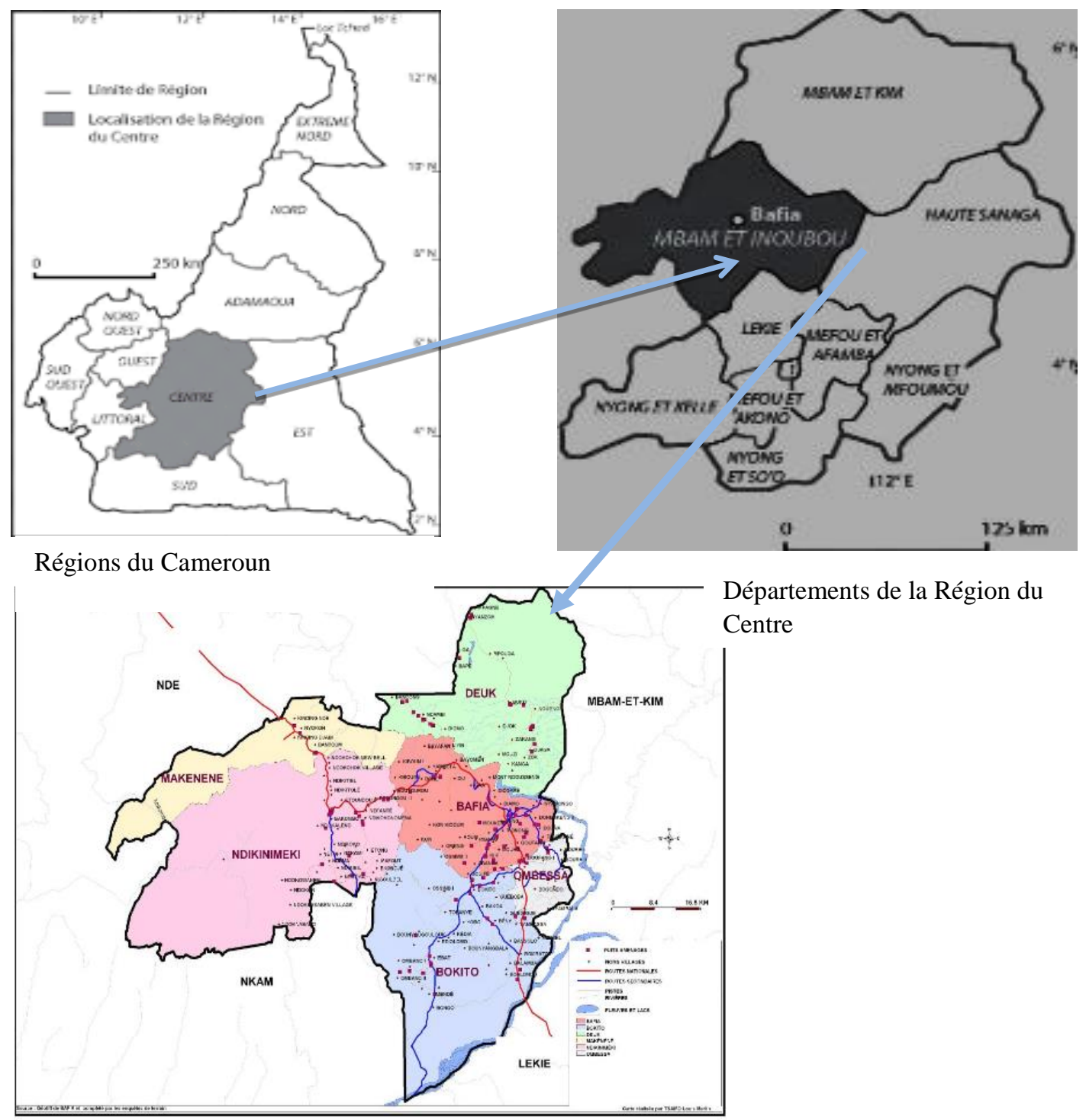

Arrondissements du Mbam et Inoubou

Figure 1: Localisation de la zone d'étude (adapté de Tsamo, 2008).

\section{RESULTATS}

\section{Caractéristiques socio-économiques de} l'activité piscicole

\section{Répartition des pisciculteurs en fonction des} arrondissements

La répartition des pisciculteurs en fonction des arrondissements du département du Mbam et Inoubou (Figure 2) permet de constater que les arrondissements de Bafia et de Kiiki ont la moitié des pisciculteurs et que les cinq (05) autres se partagent l'autre moitié; dont Kon est l'arrondissement ayant le moins de pisciculteurs.

Répartition des étangs par arrondissement

La répartition des étangs par arrondissement (Figure 3) révèle que Kiiki compte plus du quart des étangs recensés, suivi de Bafia. Tandis que Bokito et Nitoukou représentent à eux deux moins d'un huitième. 
Répartition des pisciculteurs en fonction de l'âge, du sexe, du statut matrimonial et du nombre d'enfants en charge

La répartition des pisciculteurs en fonction de l'âge, du sexe, du statut matrimonial et du nombre d'enfants en charge (Tableau 1) montre que l'activité piscicole dans le département est dominée à près de la moitié par des individus de la tranche d'âge de [50-60[ans, suivie par ceux de 60 ans et plus. Tandis que les tranches d'âge [30-40[et [4050 [représentent à peine plus du quart. De plus, les hommes s'investissent plus dans le secteur que les femmes qui ne représentent que 5\%. Les individus mariés représentent $90 \%$ des pisciculteurs et un peu plus de la moitié a] 3-6] enfants en charge.

Répartition des pisciculteurs en fonction de la religion, de l'ethnie, et du niveau d'étude

$\mathrm{La}$ répartition des pisciculteurs en fonction de la religion, de l'ethnie et du niveau d'étude (Tableau 2) ressort que près de la moitié des pisciculteurs est de religion catholique, suivi des protestants et des musulmans. L'ethnie Bafia est la plus représentée et lesBassa sont moindres. Concernant le niveau d'étude, le primaire est le moins représenté.

Répartition des pisciculteurs en fonction de la profession, de la formation supplémentaire et lieu de cette formation

$\mathrm{La}$ répartition en fonction de la profession, de la formation supplémentaire et du lieu de cette formation (Tableau 3) ressort que les pisciculteurs de la zone sont majoritairement cultivateurs $(37,50 \%)$, puis les entrepreneurs et les retraités avec respectivement $22,50 \%$ et enfin les fonctionnaires 17,50\%. Quant à la formation, elle est dominée à $42,50 \%$ par des domaines autres ; suivi respectivement par l'entreprenariat agropastoral (27,50\%), l'agriculture et l'élevage ( $12,50 \%$ chacun) et la pisciculture qui ne représente que $5 \%$. Les pisciculteurs reçoivent majoritairement leur formation supplémentaire dans les écoles paysannes (27,50\%), les écoles conventionnelles et les conférences/colloques à $22,50 \%$ chacun et à $20 \%$ dans les programmes.
Répartition des pisciculteurs en fonction de la principale source de revenus, du revenu mensuel moyen et de la place de la pisciculture dans leurs activités

La répartition en fonction de la principale source de revenus, du revenu mensuel moyen et de la place de la pisciculture dans leurs activités (Tableau 4) permet de constater que les activités agricoles et les affaires constituent à égalité les principales sources de revenus pour les pisciculteurs, suivies respectivement par les pensions et les salaires. Pour le revenu mensuel moyen, $32,50 \%$ des pisciculteurs ont un revenu supérieur à $200000 \mathrm{~F}$ CFA, puis suivi respectivement de ceux dont le revenu est compris entre]110-200] et [20-50]. La pisciculture constitue une activité du plan inférieur dans la zone.

Répartition des pisciculteurs en fonction du statut de la structure, du domaine, de l'objectif de production et de l'ancienneté de la structure

La répartition en fonction du statut de la structure, du domaine, de l'objectif de production et de l'ancienneté de la structure (Tableau 5) nous montre que plus des trois quarts des structures sont individuelles. Les GIC et les projets ne représentant que moins du quart des structures de production. Les structures sont à $72,50 \%$ installées sur les domaines familiaux et à $20 \%$ sur les domaines achetés. L'objectif de production principal est l'autoconsommation. Concernant l'ancienneté, la moitié des structures a vu le jour entre 20002010 , et $45 \%$ après. Ainsi les pisciculteurs ont une expérience de moins de 20 ans.

Répartition des pisciculteurs en fonction de la main d'oeuvre, du salaire par individu et du prix de vente par espèce

La répartition des pisciculteurs en fonction de la main d'œuvre, du salaire par individu et prix de vente par espèce (Tableau 6) ressort que la main d'œuvre utilisée dans la production piscicole reste à $45 \%$ salariale ; $80 \%$ des structures n'ont pas pu se prononcer sur le volet salarial et la même proportion sur le prix de vente des espèces produites. Pour quelques rares informations, 7,50\% de pisciculteurs disent vendre le kilogramme de 
tilapia à $1500 \mathrm{~F} \mathrm{CFA,} \mathrm{celui} \mathrm{du} \mathrm{silure} \mathrm{à} 2000$ 2300 F CFA (2,50\%). Le prix est fixé non seulement en fonction de l'espèce, mais aussi du lieu de vente.

\section{Caractéristiques zootechniques}

Répartition des pisciculteurs en fonction du type d'étang, du type de culture, du type de pisciculture et de la structure de production

La répartition en fonction du type d'étang, du type de culture, du type de pisciculture et de la structure de production (Tableau 7) nous montre que la majorité des étangs sont en déblais, une faible proportion en dérivation et pas d'étang de barrage. La polyculture se pratique en grande partie et un peu plus du quart pour la monoculture. La pisciculture simple représente $87,50 \%$ et celle intégré $7,50 \%$. Les structures de production sont principalement des étangs.

Répartition des pisciculteurs en fonction de l'espèce produite, de la conception des digues et de l'apport en eau dans les étangs

La répartition des pisciculteurs en fonction de l'espèce produite, de la conception des digues et de l'apport en eau dans les étangs (Tableau 8) ressort que l'espèce de poisson la plus produite reste les silures, puis dans l'ordre d'importance respective; les tilapias, les carpes, les kanga et poissons vipères. Les étangs sont pour la plupart mal construits avec des digues verticales. L'alimentation en eau des étangs se fait principalement par les eaux souterraines (nappes phréatiques).

\section{Répartition des pisciculteurs en fonction de la provenance des premiers alevins, du type de reproduction, de l'alimentation et de la fréquence d'alimentation}

La répartition en fonction de la provenance des premiers alevins, du type de reproduction, de l'alimentation et de la fréquence d'alimentation (Tableau 9), permet de voir que plus de la moitié des pisciculteurs se ravitaillent en alevins pour la première fois auprès des projets, $37,50 \%$ auprès d'autres producteurs et commerçants et le reste en milieu naturel. Aucune structure ne pratique la reproduction artificielle, le renouvellement des alevins se fait par achat. Le type d'alimentation reste de moitié la fertilisation, suivi respectivement par les aliments composés locaux et importés. Quant à la fréquence d'alimentation, elle est de $32,50 \%$ de deux fois/jour, de $25 \%$ momentanément et tous les deux jours respectivement.

Répartition des pisciculteurs en fonction du nombre d'étangs, de la superficie de l'étang, de la structure de vidange et de l'ensoleillement

La répartition en fonction du nombre d'étangs, de la superficie de l'étang, de la structure de vidange et de l'ensoleillement (Tableau 10) montre que les pisciculteurs ayant un nombre d'étangs compris entre]0-4] représentent les $3 / 4$ de l'effectif ; ceux ayant un nombre d'étangs compris entre]4-8] un peu plus du quart.

Les étangs dont la superficie est inférieure ou égale à $100 \mathrm{~m}^{2}$ constitue 40,97\% de l'effectif des étangs, ceux de]100-400] représentent près du quart, 19,44\% pour ceux de]400-800] et à peu près la même proportion pour les étangs de plus de $800 \mathrm{~m}^{2}$; dont $12,50 \%$ de plus de $1000 \mathrm{~m}^{2}$.

Par ailleurs, plus de la moitié des étangs n'ont pas de structures de vidange et pour ceux qui en possèdent, elles sont en majorité en tuyaux PVC. Les étangs sont en majorité bien ensoleillés.

\section{Répartition des pisciculteurs en fonction de l'état de la structure et l'orientation de la production}

La répartition en fonction de l'état de la structure et de l'orientation de la production (Figure 4) permet de constater que près de $3 / 4$ des étangs étaient inactifs au moment de notre passage, parmi lesquels $28,30 \%$ étaient secs. Quant à l'orientation de l'activité, elle reste dédiée au grossissement. Aucune structure n'est orientée vers l'alevinage, mais la reproduction se passe naturellement dans les étangs et permet ainsi de contribuer au renouvellement des animaux.

\section{Caractéristiques sanitaires}

Des caractéristiques sanitaires (Tableau 11), il ressort que presque tous les pisciculteurs n'utilisent pas les pesticides (herbicides) pour le nettoyage des alentours des étangs. Néanmoins, certains témoignent avoir utilisé 
au moins une fois. De plus, elles permettent de s'en rendre compte que la majorité des étangs ne sont pas protégés.

Quant au défrichage des étangs, la moitié des producteurs gardent toujours dans un état propre leurs structures contre l'autre moitié qui malheureusement n'y arrive pas. Et nous avons fait le constat lors de notre passage. Ce qui fait que le taux de mortalité reste méconnu et non maîtrisé pour de nombreux pisciculteurs. Le taux de mortalité due aux prédateurs a été le plus élevé.

\section{Contraintes liées à l'activité piscicole dans la zone}

Les contraintes liées à l'activité piscicole dans la zone (Tableau 12) montrent que plus de la moitié des pisciculteurs ne connaissent pas l'existence des projets dans le domaine. La même proportion ne sont pas connus des services compétents. Aucun pisciculteur n'a accès au crédit et $85 \%$ n'appartiennent à aucune organisation. Les $3 / 4$ disent ne pas bénéficier de l'encadrement et de l'accès aux alevins. De plus, le vol constitue une autre contrainte déterminante et est à l'origine de plus de la moitié des pertes dans les exploitations (Figure 5).

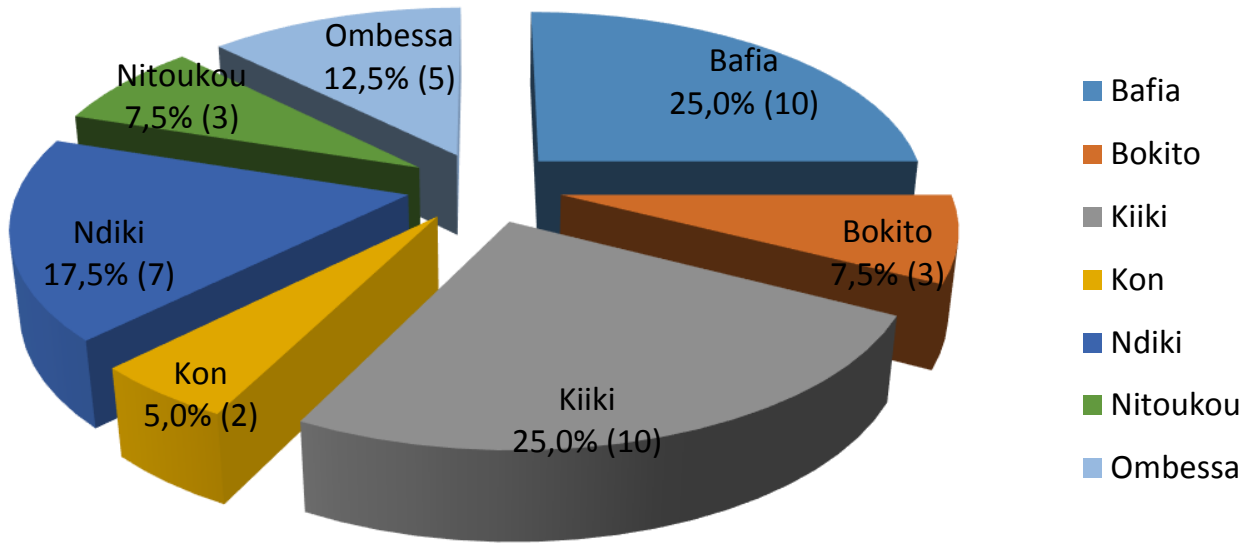

( ) Effectif de pisciculteurs par arrondissement

Figure 2 : Répartition des pisciculteurs en fonction des arrondissements.

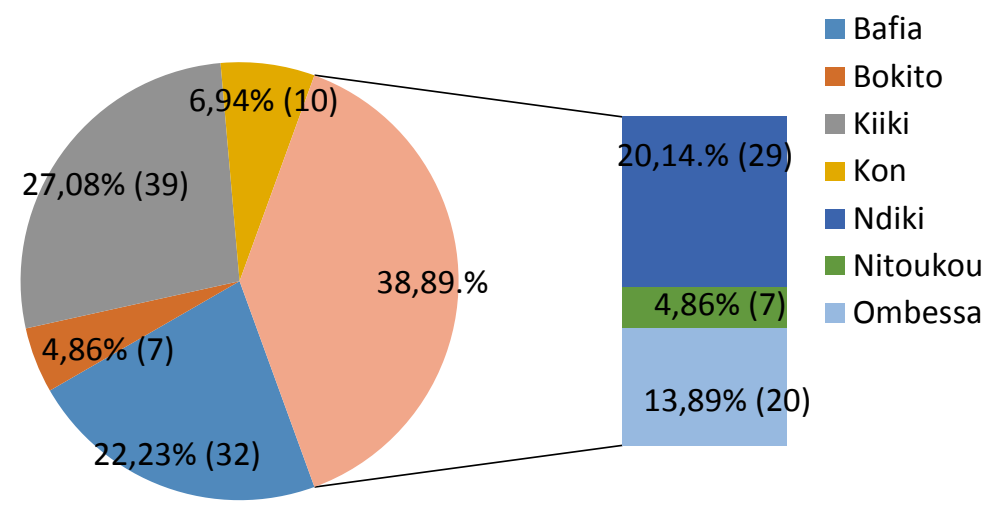

( ) Effectif d'étangs par arrondissement

Figure 3 : Nombre d'étangs par arrondissement dans le Département du Mbam et Inoubou. 
Tableau 1 : Répartition des pisciculteurs en fonction de l'âge, du sexe, du statut matrimonial et du nombre d'enfants en charge.

\begin{tabular}{lc}
\hline Caractéristiques & Fréquences(\%) \\
\hline Âges (années) & 2,50 \\
[30-40[ & 25,00 \\
[40-50[ & 40,00 \\
[50-60[ & 32,50 \\
60 et plus & \\
\hline Sexe & 95,00 \\
Masculin & 5,00 \\
Féminin & \\
\hline Statut matrimonial & 90,00 \\
Mariés & 7,50 \\
Séparation de corps & 2,50 \\
Veuve & \\
& \\
\hline Nombre d'enfants en charge & 22,50 \\
] 0-3] & 52,50 \\
] 3-6] & 17,50 \\
6-9] & 7,50 \\
Autres (animaux de compagnie) & \\
\hline
\end{tabular}

Tableau 2 : Répartition des pisciculteurs en fonction de la religion, de l'ethnie et du niveau d'étude.

Caractéristiques $\quad$ Fréquences (\%)

\begin{tabular}{lc}
\hline Religion & \\
Animiste & 5,00 \\
Catholique & 45,00 \\
Protestante & 30,00 \\
Musulmane & 17,50 \\
Témoin de Jéhovah & 2,50 \\
\hline Ethnie & \\
Bafia & 42,50 \\
Bamiléké & 7,50 \\
Banen & 27,50 \\
Bassa & 2,50 \\
Yambassa & 20,00 \\
\hline Niveau d'études & \\
Primaire & 7,50 \\
Secondaire & 40,00 \\
Supérieur & 52,50 \\
\hline
\end{tabular}


Tableau 3: Répartition des pisciculteurs en fonction de la profession, de la formation supplémentaire et du lieu de cette formation.

\begin{tabular}{lc}
\hline Caractéristiques & Fréquences (\%) \\
\hline Profession & \\
Cultivateurs & 37,50 \\
Entrepreneurs & 22,50 \\
Fonctionnaires & 17,50 \\
Retraités & 22,50 \\
\hline Formation supplémentaire & \\
Agriculture & 12,50 \\
E. A. P. (Entreprenariat Agropastorales) & 27,50 \\
Elevage & 12,50 \\
Pisciculture & 5,00 \\
Autres (maçonnerie, menuiserie...) & 42,50 \\
\hline Lieu de cette formation & \\
Ecoles conventionnelles & 22,50 \\
Ecoles paysannes & 27,50 \\
Conférences/Colloques & 22,50 \\
Programmes & 20,50 \\
Autres (cours du soir, formation continu...) & 7,50 \\
\hline
\end{tabular}

Tableau 4 : Répartition des pisciculteurs en fonction de la principale source de revenus, du revenu mensuel moyen et de la place de la pisciculture dans leurs activités.

\begin{tabular}{lc}
\hline Caractéristiques & Fréquences $\mathbf{( \% )}$ \\
\hline Principale source de revenus & 37,50 \\
Activités agricoles & 37,50 \\
Affaires & 2,500 \\
Commerce & 12,50 \\
Pension & 10 \\
Salaire & \\
& \\
\hline Revenu mensuel moyen (en milliers de francs) & 20 \\
{$[20-50]$} & 17,50 \\
] 50-80] & 7,50 \\
] 80-110] & 22,50 \\
] 110-200] & 32,50 \\
$>200$ & \\
& \\
\hline Place de la pisciculture & 7,50 \\
Activité principale & 37,50 \\
Activité secondaire & 25 \\
Activité tertiaire & 30 \\
Dérisoire & \\
\hline
\end{tabular}


Tableau 5 : Répartition des pisciculteurs en fonction du statut de la structure, du domaine, de l'objectif de production et de l'ancienneté.

\begin{tabular}{lc}
\hline Caractéristiques & Fréquences $\mathbf{( \% )}$ \\
\hline Statut de la structure & \\
Individuel & 85 \\
GIC & 12,50 \\
Projet & 2,50 \\
\hline Domaine de la structure Achat & \\
Familial (lègue) & 20 \\
Don & 72,50 \\
& 7,50 \\
\hline Objectif de production & \\
Autoconsommation + don & 37,50 \\
Commercialisation + don & 5 \\
Les deux & 57,50 \\
\hline Ancienneté (années) & \\
$\geq 50$ & 2,50 \\
20 - 50 & 2,50 \\
10 - 20 & 50 \\
$\leq 10$ & 45 \\
\hline GIC : Groupes d'Initiatives Communes &
\end{tabular}

Tableau 6: Répartition des pisciculteurs en fonction de la main d'œuvre, du salaire par individu et du prix par espèce.

\begin{tabular}{lcc}
\hline Caractéristiques & Fréquences (\%) \\
\hline Main d'œuvre & \\
Familiale & & 32,50 \\
Salariale & 45 \\
Familiale + salariale & 22,50 \\
\hline Salaire par individu (F CFA) & \\
30000 & & 5 \\
35000 & & 5 \\
40000 & & 7,50 \\
45000 & & 2,50 \\
Autre (volontariat ou par & & 80 \\
compensation) & Prix & \\
\hline Espèces & $(\mathrm{F} / \mathrm{kg})$ & $2,50-2,50$ \\
Silure & $2000-$ & $0-7,50$ \\
Tilapia & 2300 & $0-2,50$ \\
Carpe & $1000-$ & 2,50 \\
Kanga & 1500 & 2,50 \\
Poisson vipère & $1000-$ & 80 \\
Autres (espèces de poissons & 1500 & \\
sauvages) & 2300 & \\
& 2000 & \\
\hline
\end{tabular}


Tableau 7 : Répartition des pisciculteurs en fonction du type d'étang, du type de culture, du type de pisciculture et de la structure de production.

\begin{tabular}{lc}
\hline Caractéristiques & Fréquences (\%) \\
\hline Type d'étang & \\
Déblais & 82,50 \\
Dérivation & 17,50 \\
\hline Type de culture & \\
Monoculture & 30 \\
Polyculture & 70 \\
\hline Type de pisciculture & \\
Intégrée & 7,50 \\
Simple & 87,50 \\
Les deux & 5 \\
\hline Structure de production & \\
Etangs & 97,50 \\
Bacs & 2,50 \\
\hline
\end{tabular}

Tableau 8: Répartition des pisciculteurs en fonction de l'espèce élevée, de la conception des digues et de l'apport en eau dans les étangs.

\begin{tabular}{lc}
\hline Caractéristiques & Fréquences (\%) \\
\hline Espèces produites & \\
Silure (Clarias gariepinus) & 36,14 \\
Tilapia (Oreochromis niloticus) & 27,71 \\
Carpe (Cyprinus carpio) & 14,40 \\
Kanga (Heterotis niloticus) & 10,84 \\
Poisson vipère (Parachanna obscura & 10,84 \\
\hline Conception des digues & \\
Digues verticales & 82,05 \\
Digues obliques & 17,95 \\
\hline Apport en eau dans les étangs & \\
Eaux souterraines & 82,50 \\
Canal & 17,50 \\
\hline
\end{tabular}

Tableau 9: Répartition des pisciculteurs en fonction de la provenance des premiers sujets, du type de reproduction, du type d'alimentation et de la fréquence d'alimentation.

\begin{tabular}{lc}
\hline Caractéristiques & Fréquences \% \\
\hline Provenance des premiers alevins & \\
Projet & 52,50 \\
Milieu naturel & 10 \\
Autres (dons, importés) & 37,50 \\
\hline Type de reproduction & 100 \\
Naturelle & \\
\hline
\end{tabular}




\section{Type d'alimentation}

Aliment composé local

17,50

Aliment composé importé

17,50

Fertilisation

50

Autres (déchets domestiques ou d'agriculture

15

\section{Fréquence d'alimentation}

Tous les 2 jours

2 fois par jour

1 fois par jour

Tableau 10: Répartition des pisciculteurs en fonction du nombre d'étangs, de la superficie des étangs, de la structure de vidange et de l'ensoleillement.

\begin{tabular}{|c|c|}
\hline Caractéristiques & Fréquences $(\%)$ \\
\hline \multicolumn{2}{|l|}{ Nombre d'étangs } \\
\hline ] 0-4] & 75 \\
\hline ] 4-8] & 17,50 \\
\hline ] 8-11] & 5 \\
\hline$>11$ & 2,50 \\
\hline \multicolumn{2}{|c|}{ Superficie de l'étang $\left(\mathbf{m}^{2}\right)$} \\
\hline$\leq 100$ & 40,97 \\
\hline ] 100-400] & 21,53 \\
\hline ] 400-800] & 19,44 \\
\hline ] 800-1000] & 5,56 \\
\hline$>1000$ & 12,50 \\
\hline \multicolumn{2}{|c|}{ Structure de vidange Moine } \\
\hline Tuyau PVC & 2,50 \\
\hline En terre & 40 \\
\hline \multirow[t]{2}{*}{ Absente } & 5 \\
\hline & 52,50 \\
\hline \multicolumn{2}{|l|}{ Ensoleillement } \\
\hline Bien ensoleillé & 87,50 \\
\hline Peu ensoleillé & 12,50 \\
\hline
\end{tabular}

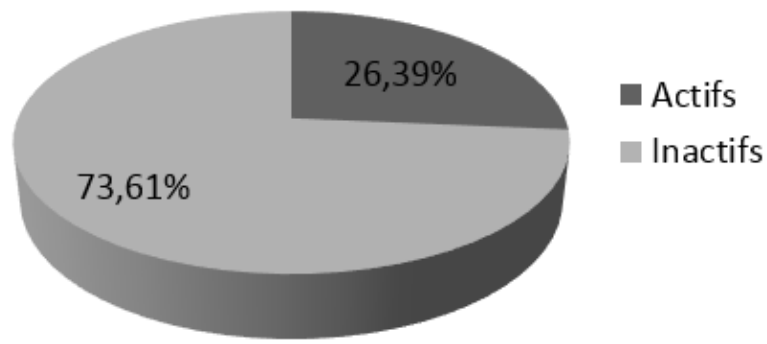

Figure 4 : Répartition des étangs en fonction de leur état. 
Tableau 11 : Répartition des pisciculteurs en fonction de l'utilisation ou non des pesticides, de la protection ou non des étangs, de la régularité du défrichage, du taux de mortalité et des causes de mortalité.

\begin{tabular}{lc}
\hline Caractéristiques & Fréquences (\%) \\
\hline Utilisation des pesticides & 5,13 \\
Oui & 94,87 \\
Non & \\
\hline Protection des étangs & 12,82 \\
Oui & 87,18 \\
Non & \\
\hline Défrichage des étangs & 50 \\
Toujours & 50 \\
Pas toujours & \\
\hline Mortalités & 12,50 \\
Très peu & 30 \\
Peu & 5 \\
Moyen & 7,50 \\
Elevé & 45 \\
Ne sait pas & \\
\hline Causes de mortalité & 42,50 \\
Prédateurs & 25 \\
Causes zootechniques & 32,50 \\
Ne sais pas &
\end{tabular}

Tableau 12 : Répartition des pisciculteurs en fonctions des contraintes liées à l'activité piscicole.

\begin{tabular}{lc}
\hline Caractéristiques & Fréquences (\%) \\
\hline Connaissance des projets & 32,50 \\
Oui & 67,50 \\
Non & \\
\hline Enregistrement auprès des services compétents & 32,50 \\
Oui & 57,50 \\
Non & 10 \\
Pas encore & \\
\hline Sources de financement & 85 \\
Personnelle & 0 \\
Crédit & 15 \\
Subvention & \\
\hline Appartenance à une organisation & 15 \\
Oui & 85 \\
Non & \\
\hline Accessibilité aux ressources technique et & \\
génétique & 10 \\
Manque d'alevins & 15 \\
Manque d'encadrement technique & 75 \\
Les deux combinés & \\
\hline
\end{tabular}




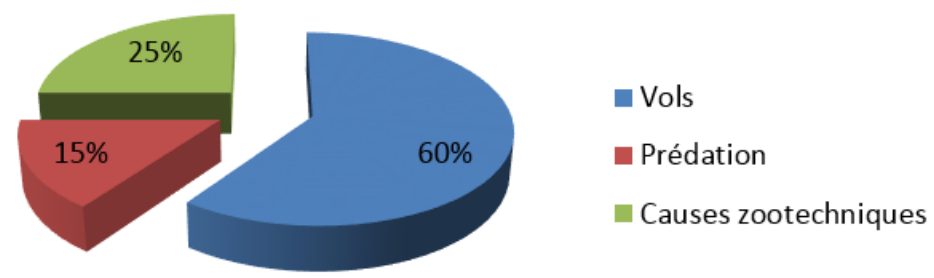

Figure 5 : Causes majeures des pertes.

\section{DISCUSSION}

\section{Caractéristiques socioéconomiques}

Quarante (40) structures piscicoles ont contribué à la réalisation de ce travail dans le département du Mbam et Inoubou. Ceci corrobore avec l'effectif exploité par Bomba (2016) dans le département du Mfoundi (Centre-Cameroun); mais est en nette distension avec celui exploité par Hirigohen et al. (1997), Pouomogne et Pemsl (2008) respectivement dans les départements de la Mefou (Afamba et Akono) (Centre-Cameroun) et du Noun (Ouest-Cameroun) qui était de 62. Ce faible effectif s'expliquerait par une considération socio-culturelle. En effet ces populations s'attachent plus aux activités agricoles; et quand bien même elles pensent à l'élevage, il s'agit du gros et du petit bétail. La pisciculture est en cours de décollage dans ce département.

La distribution des pisciculteurs reste relativement générale, ainsi deux arrondissements concentrent près de la moitié des producteurs. Ce résultat s'oppose à celui de Bomba (2016) où plus de cette proportion $(57,50 \%)$ revenait au seul arrondissement de Yaoundé 4. De plus quant à l'ancienneté, elle confirme les résultats de $97,78 \%$ rapportés par Bomba (2016). Cette récente prise de place de la pisciculture trouverait son explication non seulement à la diversification des productions, et partant des sources de revenus par les populations, mais aussi aux efforts de vulgarisations entrepris ces dernières années par les pouvoirs publics et les partenaires au développement (MINEPIA, 2015).
La pisciculture reste une activité secondaire $(7,5 \%)$ dans ce Département, pratiquée majoritairement par les agriculteurs $(62,5 \%)$. Ce résultat est similaire à celui trouvé au Cameroun dans le Département de la Mefou par Hirigoyen et al. (1997), en République Démocratique du Congo par Ngandi (2009) qui concluent que $77 \%$ de ses enquêtés s'occupaient principalement aussi de l'agriculture. Toutefois, ces résultats sont très différents de ceux de Kpenavoun et al. (2017) qui rapportent qu'au Bénin la pisciculture était l'activité principale jusqu'à $32,5 \%$ de la population. Ces résultats démontrent ainsi que la pisciculture a plus décollée au Bénin qu'au Cameroun.

Les résultats sur la tranche d'âge, le genre, le statut matrimonial, le niveau d'étude et l'appartenance religieuse et ethnique divergent avec ceux de Hirigoyen et al (1997) dans le Département de la Mefou où les pisciculteurs étaient tous chrétiens et de sexe masculin. De même avec ceux de Pouomogne et Pemsl ( 2008) dans le Département du Noun où $80 \%$ des pisciculteurs avaient entre 30 et 50 ans, et étaient majoritairement musulmans. Et de ceux de Kpenavoun et al. (2017) au Bénin où le niveau d'étude supérieur était moins intéressé par cette activité (15\%). Par contre ils convergent avec ceux de Bomba (2016) dans le Mfoundi où les pisciculteurs étaient à 90\% des hommes, 92,5\% chrétiens, $77,5 \%$ mariés, le niveau d'étude universitaire dominait à 42,5\% et une ethnie majoritaire qui était les Bétis. Toutefois, Bomba (2016) a relevé que $82,5 \%$ étaient âgés entre 36 et 65 
ans. La différence de tranche d'âge serait liée au fait que la plupart des propriétaires ont un certain niveau de revenus; celle religieuse se rattacherait plus au fait socioculturel et celle de sexe s'expliquerait par l'émancipation de plus en plus constatée des femmes. Cela corrobore avec les résultats trouvés par Yao et al. (2017) en Côte d'Ivoire (94\%) et ceux de Ngandi (2009) dans le village Mobi et ses environs (75,5\%) qui avaient démontré que cette activité était majoritairement effectuée par les hommes

Le statut des structures, la source de financement rejoignent les résultats de Bomba (2016) qui dans le Mfoundi stipulaient que $80 \%$ des pisciculteurs étaient des particuliers, 97,5\% des structures piscicoles étaient montées sur fonds personnels. Par contre, la domination des cultivateurs dans le secteur s'oppose à ses résultats qui rapportaient que le secteur était dominé par les retraités à $37,5 \%$. Le caractère dominant des fonds personnels sur la mise en place de l'activité piscicole dans la zone serait tributaire à l'absence des structures bancaires capables de financer les activités agropastorales, et quand lorsqu'elles existent, les mécanismes d'accès restent lourds. De plus, la domination des cultivateurs relèverait de ce que le Mbam et Inoubou reste essentiellement agricole par rapport au Mfoundi qui est le bastion urbain et dont les retraités se convertiraient de façon naturelle à une autre activité.

\section{Caractéristiques zootechniques}

Les résultats sur le type d'étang, l'alimentation en eau et le type de culture sont en conformité avec ceux de Bomba (2016) dans le Mfoundi où $90 \%$ des étangs dans la zone étaient en déblais et de nappes phréatiques et que la polyculture y était pratiquée à 97,5\% . Ces résultats sont contraires à ceux de Ngandi (2009) dans le village Mobi et ses environs en République Démocratique du Congo où les étangs de barrage étaient dominant à 72,5\% . Par ailleurs, ils contredisent ceux de Bomba (2016) quant à l'espèce la plus cultivée et au nombre d'étangs par individu qui étaient respectivement le tilapia $(32,74 \%)$ et de 4 à 6 étangs (35\%). Cette opposition de l'espèce la plus cultivée serait due au fait que les populations du Mbam et Inoubou apprécieraient plus la consommation du silure. Ils sont par ailleurs en conformité avec ceux de Ngandi (2009) en République Démocratique du Congo. Par contre, la convergence observée sur la pratique de la polyculture malgré la différence revue à la baisse témoignerait de l'importance accordée à l'association des espèces. Mais cette association reste sans doute non maîtrisée; car ne tient ni compte des espèces, ni des densités et tailles de mise en charge des étangs.

Les résultats concernant la situation des étangs, l'approvisionnement en alevins, ne corroborent pas avec ceux de Bomba (2016) qui dans le Mfoundi rapportait que $80,87 \%$ des étangs fonctionnaient et $47,5 \%$ des alevins venaient du milieu naturel. En revanche la domination de l'autoconsommation pour ce qui est de la destination des productions va de convergence avec ses résultats $(60,67 \%)$. La provenance remarquée des alevins auprès des milieux professionnels s'expliquerait par la vulgarisation des techniques de propagation artificielle mises au point pour produire en masse des juvéniles dans les nombreuses stations piscicoles du pays pour résoudre le problème de manque d'alevins (Nguenga, 2000 ; Nguenga et al., 2000). Toutefois, ces techniques sont restées au niveau des projets car les pisciculteurs ignorent leur pratique.

\section{Caractéristiques sanitaires et contraintes}

Les caractéristiques sanitaires permettent de constater que $87,18 \%$ des étangs ne sont pas protégés, la moitié des pisciculteurs n'assure pas toujours le nettoyage des étangs, ce qui entrainerait des mortalités.

Quant aux contraintes, nombreux d'entre les pisciculteurs ne sont pas informés de l'existence des projets dans leur secteur d'activité, de même ils ne sont pas connus par 
l'autorité compétente, et n'ont pas accès aux crédits et subventions. Selon Kenfack et al. (2019), ces contraintes ne sont pas spécifiques à cette zone mais concernent tout le pays. Tout comme à Mobi et ses environs en République Démocratique du Congo (Ngandi, 2009), le manque de moyen financier et d'organisation, le manque d'appui technique et le vol fréquent constituent les principales contraintes de l'essor de cette activité.

\section{Conclusion}

Au terme de cette étude dont l'objectif était de caractériser l'activité piscicole dans le Département du Mbam et Inoubou, il en ressort que : Sur le plan socio-économique, l'ethnie Bafia domine le secteur et la religion chrétienne reste prépondérante parmi les pisciculteurs. Ces éleveurs sont des hommes mariés avec plusieurs enfants à charge, la main d'œuvre reste à la fois salariale et familiale pour cette activité. Le niveau de formation dans le domaine piscicole reste quasi nul. Les principales sources de revenus sont les activités agricoles et les affaires au sein des acteurs piscicoles. Sur le plan technique, les étangs en déblais de nappes phréatiques constituent les principales structures de production piscicole, dans lesquels une polyculture de type artisanale reste en grande partie pratiquée. Cependant, ces étangs connaissent un sérieux problème de gestion; car mal construits et peu vidangeables. Les principales espèces élevées sont les silures (Clarias gariepinus), les tilapias (Oreochromis niloticus), les carpes (Cyprinus carpio), les kanga (Heterotis niloticus) et les poissons vipère (Parachana obscura). L'alimentation est pour la majorité assurée par la fertilisation des étangs et l'aliment composé. L'approvisionnement en alevins se fait au niveau des projets, ce qui entraîne des problèmes d'accessibilité et de proximité d'où la manne des intermédiaires qui abusent les pisciculteurs, les poussant à l'abandon. Sur le plan sanitaire, bon nombre d'étangs ne sont pas protégés. De plus l'entretien de ces étangs reste un cauchemar pour les propriétaires, d'où les pertes liées aux prédateurs. Pour la plupart des pisciculteurs ce secteur d'activité est rentable et mais reste à promouvoir davantage. Les principales contraintes sont le vol, l'insuffisance d'alevins, faible accès aux subventions et crédits, absence d'organisation des acteurs et d'encadrement. Dans ce Département l'activité piscicole est encore pratiquée de manière artisanale. A l'avenir, l'état devrait accompagner les acteurs de cette activité par de multiples formations.

\section{CONFLIT D'INTERETS}

Les auteurs déclarent qu'il n'existe aucun conflit d'intérêts.

\section{CONTRIBUTIONS DES AUTEURS}

Pour la réalisation de ce travail tous les auteurs ont contribué : les auteurs TTC et BA ont défini et arrêté le thème du travail; l'auteur TTC a tracé toutes les courbes et tableaux, et a rédigé la première mouture du manuscrit, l'auteur BA a collecté et analysé les données, les auteurs KA et $\mathrm{TJ}$ ont mis à jour la revue de la littérature. Tous les auteurs ont lu et apporté des corrections à la version finale du manuscrit.

\section{REMERCIEMENTS}

Les auteurs remercient :

-La Délégation Départementale de l'Elevage des Pêches et des Industries Animales (DDEPIA) du Mbam et Inoubou qui a mis à leur disponibilité le fichier départemental des pisciculteurs, et les a assigné par correspondance aux différentes Délégations d'Arrondissements de l'Elevage de Pêches et des Industries Animales (DAEPIA) et les différents centres zootechniques et vétérinaires ;

- Le Service Technique Départementale ACEFA (PCP-ACEFA) du programme C2D qui a permis d'identifier les pisciculteurs faisant l'objet d'un encadrement par leurs agents figurant dans les registres. 


\section{REFERENCES}

Bomba OL. 2016. Caractérisation des fermes piscicoles dans la zone forestière du centre : cas du Département du Mfoundi. Mémoire d'ingénieur agronome, Faculté d'Agronomie et des sciences Agricole, Université de Dschang, Cameroun. 47pages

FAO. 2016. La situation mondiale des pêches et de l'aquaculture. Contribuer à la sécurité alimentaire et à la nutrition de tous. Rome, 224p.

FAO. 2018. La situation mondiale des pêches et de l'aquaculture. Atteindre les objectifs de développement durable. Rome. Licence : CC BY-NC-SA3.0 IGO. 254p.

Hirigoyen JP, Manjeli Y, Moucharou GC. 1997. Caractéristiques de la pisciculture dans la zone forestière du centre Cameroun. Tropicultura, 15(4) : 180185.

INS (Institut National de Statistiques). 2013. Annuaire statistique du Cameroun, chapitre 15 : Pêche et élevage. Pp 265282.

Kando G. 1995. L'agriculture urbaine en Afrique tropicale : Revue de littérature ; résultats de visites aux institutions de l'Afrique de l'Ouest et stratégie d'appui à la mise en place d'un projet de recherche régionale sur l'agriculture urbaine. Centre de Recherche pour le Développement International, Division de l'Environnement et de Ressources Naturelles, 38 pages.

Kenfack A A J, Ducarme C, Micha J-C. 2019. La pisciculture au Cameroun: bilan et perspectives. Int. J. Biol. Chem. Sci., 13(2): 1140-1161. DOI: https://dx.doi.org/10.4314/ijbcs.v13i2.44 Kpenavoun SC, Gandonou E, Adegbidi A,

Abokini E. 2017. Mesure et déterminants de l'efficacité technique des pisciculteurs du Bénin. Int. J. Biol. Chem. Sci., 11(5): 2194-2208. DOI :

https://dx.doi.org/10.4314/ijbcs.v11i5.20
Kouam J. 2004. Projets de développement piscicole et la vulgarisation agricole au Cameroun. Pp. 21-29 in Aquaculture extension in Sub-Saharan Africa. FAO Fisheries Circular (1002). Rome, FAO.

MINEPIA. 2015. Rapport d'activités : Le Cameroun multiplie des initiatives dans l'aquaculture pour booster sa production de poissons - Investir au Cameroun, 25p.

Ngandi LN. 2009. Caractérisation technique et socio-économique de l'exploitation piscicole du village Mobi et ses environs. Rapport de stage d'Ingénieur en Agronomie, Institut facultaire des sciences agronomiques de yangambi «IFA-YANGAMBI » Republique democratique du congo. 46 Pages

Nguenga D, Teugels GG, Ollevier F. 2000. Fertilization, hatching, survival and growth rates in reciprocal crosses of two strains of an African catfish Heterobranchus longifilis Valenciennes 1840 under controlled hatchery conditions. Aquaculture Research, 31: 565-573.

Nguenga D. 2000. Partial gonadectomy in the catfish Heterobranchus longifilis (Teleostei, Clariidae): Regeneration time, quality and quantity of postsurgical sperm production. The Israeli Journal of Aquacuture-Bamidgeh, 52(4): 167-172.

Pouomogne V, Pemsl DE. 2008. Recommendation domains for pond aquaculture. Country Case study: Development and status of freshwater aquaculture in Cameroon- Worldfish Center Studies and Reviews $\mathrm{N}^{\circ} 1871$, The WorldFish Center (Ed.), Penang (Malaysia), 60p.

Teleu NE, Ngatchou A. 2006. Première évaluation $\mathrm{du}$ secteur avicole au Cameroun; structure et importance du secteur avicole commercial et familial pour une meilleure compréhension de l'enjeu deaviaire. Rapport des consultants nationaux. 
Tiogué TC, Tomedi ETM, Nguenga D, Tchoumboue J. 2010. Caracteristiques de morphologie générale et de croissance du Cyprinidae africain Labeobarbus batesii dans la plaine inondable des Mbô, Cameroun. Int. J. Biol. Chem. Sci., 4(6): 1988-2000.

DOI: 10.4314/ijbcs.v4i6.64909

Tomedi E. 2015. Etat des lieux de la pisciculture au Cameroun. Hôtel Hilton à Yaoundé, l'influenza 31 mars 2015.

Tsamo LM. 2008. Intercommunalité et gestion participative de l'approvisionnement en eau potable dans le département du Mbam et Inoubou (Région du Centre). DEA en Géographie, Université de Yaoundé I. Mémoire online https://www.memoireonline.com/02/13/7 048/m_Intercommunalite-et-gestionparticipative-de-1-approvisionnement-eneau-potable-dans-le-departemen6.html.

UNCHS (United Nations of Human Settlements). 2001. Cities in a globalizing world: global report on human settlements. London: Earth scans Publications, $344 \mathrm{p}$.

Wilhelm M. 2014. Rapport de méthodes, Échantillonnage boule de neige: La méthode de sondage déterminé par les répondants. Office fédéral de la statistique (OFS). Université de Neuchâtel, Suisse. 60 pages

WORLDFISH CENTER. 2005. Le poisson et la sécurité alimentaire en Afrique. World Fish Center, Penang (Malaisie).

Yao AH, Koumi AR, Atse BC, Kouamelan EP. 2017. État des connaissances sur la pisciculture en Côte d'Ivoire. Agronomie Africaine, 29(3) : 227 - 244.

Zango P, Tomedi ETM, Efole ET, Tiogue TC, Nguenga D, Kamanke KMS, Mikolasek O, Tchoumboue J. 2016. Performances de reproduction du poisson chat endogène du Cameroun Clarias jaensis (Boulenger, 1909) en milieu contrôlé. Int. J. Biol. Chem. Sci., 10(2): 533-542. DOI : http://dx.doi.org/10.4314/ijbcs.v10i2.7. 